EPiC Series in Engineering
Volume 3, 2018, Pages 227-235
HIC 2018. 13th International
Conference on Hydroinformatics

\title{
Using compensated fluorescence probes data for proactive water treatment management
}

\author{
Edoardo Bertone ${ }^{1}$, Guilherme Franklin de Oliveira ${ }^{1}$, Rodney Stewart ${ }^{1}$ and \\ Kelvin O'Halloran ${ }^{2}$ \\ ${ }^{1}$ School of Engineering and Built Environment, and Cities Research Institute, Griffith University, \\ Parklands Drive, Southport, QLD, 4215, Australia \\ ${ }^{2}$ Seqwater, 117 Brisbane Street, Ipswich, QLD, 4305, Australia \\ e.bertonedgriffith.edu.au
}

\begin{abstract}
In this study we investigated and quantified the effects of a number of environmental conditions on the readings of fluorescent dissolved organic matter (fDOM) and total algae probes. These currently monitor fDOM, chlorophyll-a and phycocyanin for the full depth profile of different reservoirs in South-East Queensland (Australia), but interferences and quenching affecting these parameters have led to uncertainty in the reliability of the readings. Additionally, in the case of the total algae probe, obtaining reliable estimates of algal biovolume or cell counts is challenging since the pigments content varies with species and several other environmental variables influence estimates. With regards to the fDOM, a number of experiments were performed which enabled the development of a sequential compensation model accounting for the main trivial quenching. In addition, the compensated readings were compared to other experiments' outputs to check for correlations between readings and character/molecular weight of DOM to develop an accurate real-time model that may be useful in assisting DOM removal by coagulation. Preliminary work with the algae probe also showed potential to derive more specific information on species/abundance for better cyanobacteria management.
\end{abstract}

Keywords: Cyanobacteria, fDOM probe, Fluorescence, Total algae probe, Water treatment

\section{Introduction}

The removal of organic matter from the raw water is critical for drinking water treatment plants to guarantee that safe, high quality water is delivered to the consumers' taps; thus a constant monitoring of dissolved organic matter (DOM) concentration is necessary to optimize treatment processes such as 
coagulation. However, despite the fact that the knowledge of the character of the DOM would be crucial, given that specific DOM fractions have different propensity to removal and to disinfection byproducts formation, experiments for determining DOM character are expensive and time-consuming, with limited availability for real-time deployment in lakes and reservoirs. This way, simple indexes such as $\mathrm{UV}_{254}$ or dissolved organic carbon (DOC), which can only approximate the total DOM amount, are estimated on a regular basis for water treatment purposes.

Similarly, sampling cyanobacteria in reservoirs is essential for any monitoring program for optimum reservoir water quality management, albeit cyanobacteria are typically monitored at few locations and only once or twice per month due to the associated cost and time required, while temporal and spatial variations of certain species can lead to peaks not being detected through the current monitoring frequency (Pobel, Robin, \& Humbert, 2011).

In recent years there has been a widespread diffusion of remote sensing tools to monitor water quality in reservoirs, and with it, a larger deployment of fluorescence probes which, among others, can measure in-situ the amount of fluorescent dissolved organic matter (fDOM) and algae pigments such as chlorophyll-a (chl-a) and phycocyanin (PC). Probes such as the fDOM, for instance, emit a beam of ultraviolet (UV) light at a specific wavelength to excite certain organic molecules in a given sample matrix, and in turn detect the fluorescent signal at a longer wavelength emitted by the excited molecules. Despite constituting a simple, sensitive, rapid, non-invasive methodology for remote monitoring of DOM (Zamyadi, Choo, Newcombe, Stuetz, \& Henderson, 2016), the fDOM measurements are subject to quenching caused by changes in temperature, turbidity, $\mathrm{pH}$, salinity and inner filter effect (IFE). To gain higher accuracy from fDOM probes, further site-specific compensation/correction is required beyond the manufacturer's recommendations (Carstea, 2012).

In addition to such interferences, linking the chl-a and PC signals with cyanobacterial type and amount is further complicated by the fluorescence excitation spectra not being constant within each algal species, but changing based on environmental conditions (Beutler et al., 2003). Thus, although each species typically has specific chl-a and PC content per cell, usually proportional to the cell volume (Brient et al., 2008), such content can depend on the stage of cell growth, light regime, and nutrient availability to name a few. As a consequence, it has been found that the PC content per cell, for certain cyanobacteria species, is higher during the exponential growth phase (Chang, Hobson, Burch, \& Lin, 2012) or at the later growth stages (Ziegmann, Abert, Müller, \& Frimmel, 2010). High light intensities seem to reduce the PC content although there is no agreement on light effects based on a comprehensive analysis of past research (Zamyadi et al., 2016). Abundance of nutrients can also lead to an increased production of pigments (Beutler et al., 2003). Thus all these environmental factors are added to the limitations of online optical monitoring tools and resulting discrepancies between in-situ and lab experiments. However, it has been asserted that, although the intensity of the fluorescence spectrum can change due to factors outlined above, the pattern of the fluorescence spectrum does not change (Henrion, Henrion, Böhme, \& Behrendt, 1997). There is potential to conduct targeted experiments to account for such interferences and environmental factors, in order to "normalise" the pigment estimates and link them to the likelihood of the presence of specific cyanobacteria species (Bertone, Burford, \& Hamilton, 2018).

Based on previous research studies, the usual $\mathrm{pH}$ range in natural waters, between $\mathrm{pH} 5-9$, seems to have minor impact on fluorescence analysis, so it is not of major concern for lake monitoring applications. Similarly, quenching effects of metal ions are outweighed by more important environmental factors controlling DOM fluorescence. Salinity alters intramolecular reaction and interferes with fDOM readings; however, as the lakes under study are freshwater systems, their salinity levels are negligible in terms of potential quenching. In this study therefore the focus has been on calibrating the fDOM readings for temperature, turbidity and IFE, and linking the compensated fDOM readings to the prevalence of specific DOM fractions. A better understanding of the fraction of DOM measured by the fDOM probe would enable the conceptualization of an accurate coagulant dosing model, as well as a trihalomethanes (THMs) formation potential prediction model, based on 
real-time fDOM data, leading to more proactive water treatment management. With the total algae probe, measuring chl-a and PC, preliminary work has been conducted towards the final goal of linking the probe's readings with a probabilistic model inferring the likelihood of the abundance of different cyanobacteria species.

\section{Methods}

For several weeks starting from March 2017, raw water samples were collected on a weekly basis from Tingalpa Reservoir, operated by Seqwater, the main bulk water supplier in South-East Queensland (SEQ). This reservoir was the focus of this project as the nearby Capalaba water treatment plant (WTP) has had issues with organic removal, as well as taste and odour events (Bertone \& O'Halloran, 2016). The fDOM was measured using a branded fDOM probe and reported as quinine sulphate units (QSU) and relative fluorescence units (RFU). This fDOM probe has an excitation/emission pair of $365 \pm 5 \mathrm{~nm} / 480 \pm 40 \mathrm{~nm}$. Turbidity was measured with a branded Turbidity probe with output expressed in formazin nephelometric units (FNU). This turbidity sensor emits a near-infrared light source at $860 \pm 15 \mathrm{~nm}$ and detects scattering at 90 degrees of the incident light beam. Temperature was measured in ${ }^{\circ} \mathrm{C}$ units by a Conductivity \& Temperature probe, which uses a highly stable thermistor with extremely low-drift characteristics. UV absorbance at $254 \mathrm{~nm}, 365 \mathrm{~nm}$ and $480 \mathrm{~nm}$ were measured after sample filtration, using a $1 \mathrm{~cm}$ (for $\mathrm{UV}_{254}$ and $\mathrm{UV}_{365}$ ) and $4 \mathrm{~cm}$ (for $\mathrm{UV}_{480}$ ) quartz cell, on an UV-1800 UV-Visible Spectrophotometer (Shimadzu Corporation, Japan). In order to check for temperature effects, filtered $(0.45 \mu \mathrm{m})$ samples were warmed and cooled with hot water and ice, so that fDOM readings could be taken with sample temperature ranging between $5^{\circ} \mathrm{C}$ and $40^{\circ} \mathrm{C}$. A similar methodology was used in the past (Watras et al., 2011). To check for interferences due to high turbidity, a turbid solution was prepared by sampling over twenty litres of raw water after a large storm event from ex-Tropical Cyclone Debbie on 3 April 2017. The turbid mixture was gradually added into the storage cup, which caused the turbidity to gradually increase from $\sim 2 \mathrm{FNU}$ up to $\sim 483 \mathrm{FNU}$. Finally, to increase DOM concentration to check for IFE effects, a modified portable reverse osmosis (RO) machine was employed and applied to the raw water tap of the Capalaba WTP. The produced pure water was disposed and the output of the waste hose was reconcentrated for a few hours until the desired fDOM level was obtained, as measured by the probe.

All the data collected from the measurement probes were downloaded through a dedicated software program and exported onto MS Excel prior to data analysis. Data from $\mathrm{UV}_{254}, \mathrm{UV}_{365}, \mathrm{UV}_{480}$ and DOC tests were also inserted onto MS Excel. The collected dataset was pre-processed and checked for outliers and missing data. Based on data analysis, linear/nonlinear prediction models were developed to separately account for each interference; however, both a sequential modelling approach (i.e. apply separately each model one after each other) and multivariate models (i.e. account for all the interferences at once with one single model) were explored and assessed. In addition, the final models were compared with previously developed specific compensation models (Downing, Pellerin, Bergamaschi, Saraceno, \& Kraus, 2012; Watras et al., 2011).

Once the model was validated, the fDOM readings were taken for different samples collected from a number of reservoirs, i.e. 3 in Queensland and one in South Australia, with different DOM properties. The fDOM readings were compensated and compared to results of fluorescence regional integration (FRI) of excitation-emission matrixes (EEMs) using established methods (Chen, Westerhoff, Leenheer, \& Booksh, 2003). Moreover, the molecular weight (MW) distribution of the sample DOM was obtained through high-performance size exclusion chromatography (HPSEC).

Additional experiments have also been conducted to compare chl-a and PC production among different cyanobacteria species and growth stages, as well as comparisons between lab results with PC and chl-a readings with the total algae fluorescence values obtained from the fDOM probe. The 
excitation/emission wavelengths pair used for chl-a and PC measurements were, $470 \pm 15 / 680 \pm 20 \mathrm{~nm}$ and $590 \pm 15 / 680 \pm 20 \mathrm{~nm}$, respectively. The four cultures of cyanobacteria tested included the morphologically different Microcystis sp. and Cylindrospermopsis sp., as well as two other isolated cyanobacteria species from local reservoirs in SEQ. All cultures were scaled up to 2 litre cultures in standard media, with 3 replicates of each; cultures were sub-cultured at the commencement of the experiment, and all the cultures were maintained under controlled light and temperature conditions. At regular intervals during the growth period, subsamples were collected for chl-a and PC extractions. A subsample was also measured with the total algae probe. At the end of the experiment, culture samples were diluted into a range of dilutions for reading with the total algae probe and checked for nonlinearities, i.e. interferences due to high algal concentrations only (e.g. given same growth stage, species, light etc.). All the collected data will be input in a database, to be updated and completed with further experimental results, which will be used to train a model (e.g. Bayesian Network, or more appropriate one depending on data) providing the probability of the abundance of specific cyanobacteria species based on pigments data, as well as other available data and reservoir's history.

\section{Results and Discussion}

As concluded in previous studies (Watras et al., 2011), a single DOC concentration should suffice for determination of temperature effect on fDOM measurements. Therefore, in this study the investigation of temperature interference on fDOM readings was performed only using natural raw water collected at a specific time when the DOC concentration was $13 \mathrm{mg} / \mathrm{L}$. The linear regression compensation model for temperature over the range from 5 to $40^{\circ} \mathrm{C}$ yielded the following equation:

$$
f D O M_{T W}=f D O M_{m} /\left[-0.0064\left(T_{m}-T_{r}\right)+1.009\right]
$$

Where $\mathrm{T}$ is temperature $\left({ }^{\circ} \mathrm{C}\right)$ and the subscripts $r$ and $m$ stand for the reference $\left(22.5^{\circ} \mathrm{C}\right)$ and measured values, respectively. The model resulted in a near-perfect fit $\left(R^{2}>0.99\right)$, meaning that the fDOM reading can be accurately adjusted to account for temperature variations. A very similar pattern was found for $\mathrm{PC}$ readings of the total algae probe, with values at $5^{\circ} \mathrm{C}$ being 3 times higher than at $40^{\circ} \mathrm{C}$, while for chl-a readings no significant changes due to temperature variations were recorded.

Similar results were obtained for turbidity correction. As turbidity increases in the water, an increasing amount of the excitation light emitted by the fDOM sensor scatters, reducing the intensity of light available in the sampling volume to excite fDOM. As a result, the fDOM signal becomes nonlinear over a certain turbidity level (Downing et al., 2012). Consequently, in these experiments, the normalised data was fitted with a threshold autoregressive model accurately $\left(R^{2}=0.99\right)$ accounting for the presence of a linear and for a nonlinear behaviour based on background turbidity (Fig. 1). 


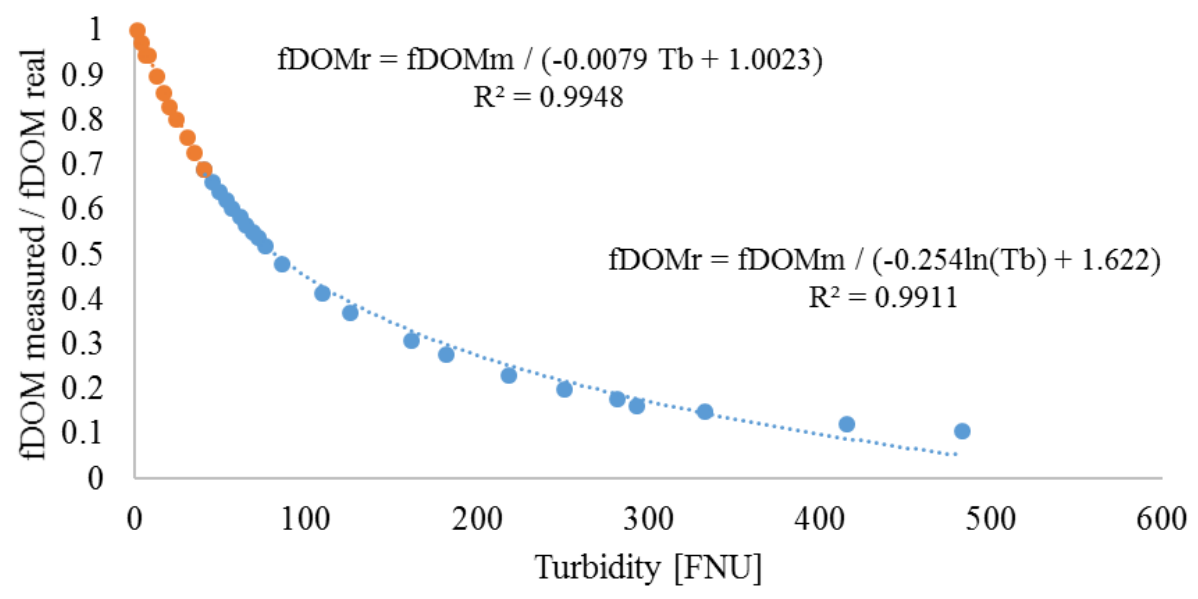

Figure 1: fDOM compensation model for turbidity interference, raw water Tingalpa reservoir

Similarly, for IFE it was noticed how this should be accounted for in the compensation model, for Tingalpa Reservoir water, any time $\mathrm{UV}_{254}$ is higher than $0.5 \mathrm{~cm}^{-1}$, as this is the threshold defining the separation between the correct, linear relationship between fDOM and DOM concentration (defined by $\mathrm{UV}_{254}$ ) and the nonlinear behaviour due to quenching (Fig. 2). Experiments were briefly conducted to also check for effects of artificial light and high salinity on fDOM readings, but as expected, there was no evident variation in readings.

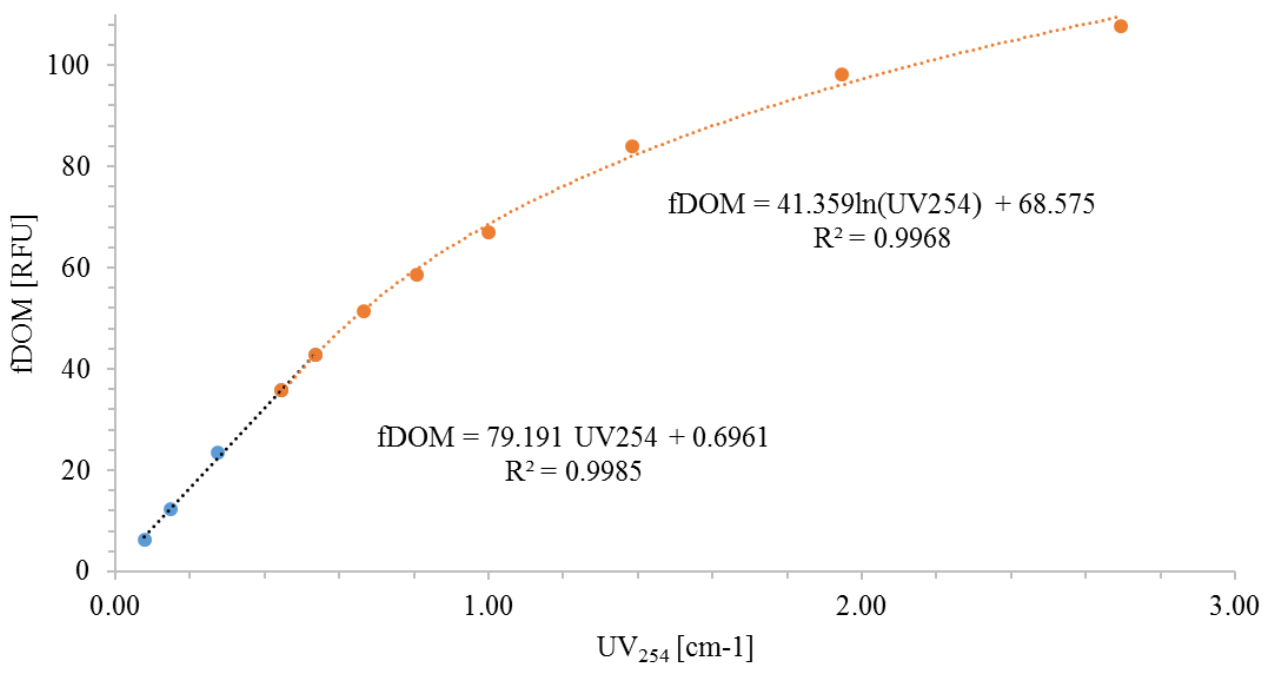

Figure 2: fDOM compensation model for IFE, raw water Tingalpa Reservoir

After the above described models were developed, a sequential adjustment model was created by applying these equations on raw fDOM data received. The ordered adjustment sequence to fDOM readings was: 1) water temperature; 2) turbidity; and 3) $\mathrm{UV}_{254}$ of each measurement. A training and test set of data were created out of the database built over time with the results of the experiments. The compensated fDOM readings were compared against the expected fDOM readings (i.e. those recorded on the experiments' days with minimum interferences, before e.g. changing temperature or increasing turbidity or DOM). The root mean squared error (RMSE) was calculated as a means of 
estimating model's performance. For comparison, a linear and a non-linear multivariate model were also developed. The performance of the models on the test set was: RMSE $=3.38 \mathrm{RFU}$ for the linear multivariate model, RMSE $=2.73 \mathrm{RFU}$ for the non-linear multivariate model, and RMSE $=0.57 \mathrm{RFU}$ for the sequential model, thus indicating that the sequential model was more accurate than the multivariate ones.

Fig. 3 and 4 clearly show the importance of correctly compensating fDOM readings for reliable DOM monitoring. Data were fragmented and not fully available, however it was enough to notice an underestimation of fDOM, particularly in times when turbidity was very high (e.g. early 2015). A correct intepretation of fDOM values can cast light on DOM behaviour in this reservoir and help to select the best intake depth for raw water extraction to the WTP, especially in cases of sudden water quality variations (e.g. following heavy rain).

Based on results from FRI and EEM, the compensated and normalised (i.e. divided by total DOC) fDOM readings were well correlated with both the fluorescence of the humic and fulvic acids' region of the spectrum. This was because all the analysed samples had a predominance on fulvic acids (see for instance Tingalpa Reservoir, Fig. 5), which resulted in a proportional "background" fluorescence intensity in the neighbouring humic acids' region. Based on this, compensated fDOM readings can be used universally to measure humic acids' abundance, however for locations where the DOM matrix does not substantially change over time (e.g. consistent predominance of fulvic acids), it seems possible to build site-specific correlations to use fDOM to measure other DOM fractions too.

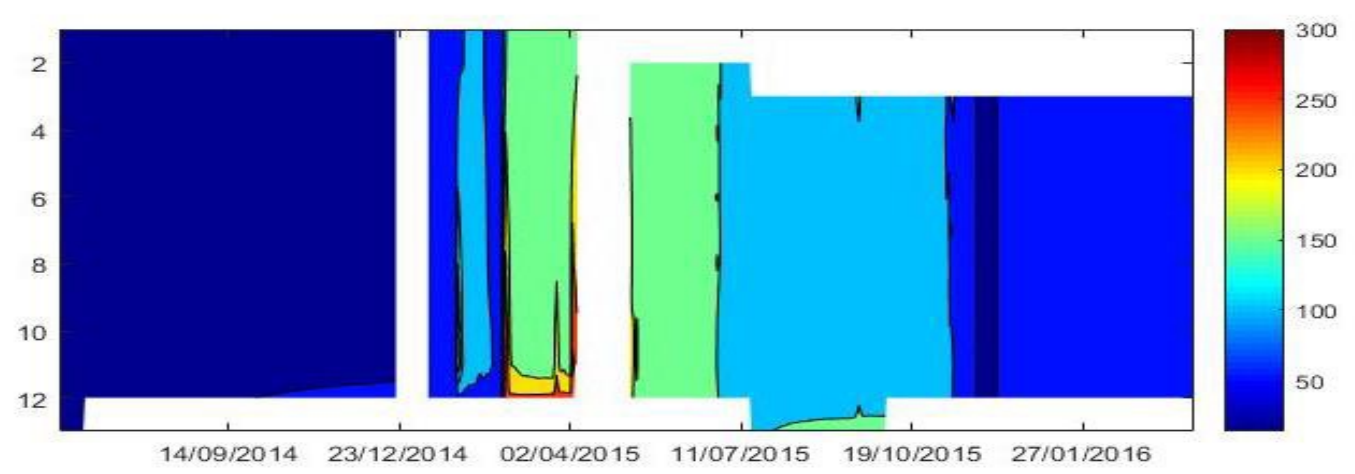

Figure 3: Compensated fDOM (RFU), Tingalpa Reservoir 2014-2016. Left-hand y axis: depth [m].

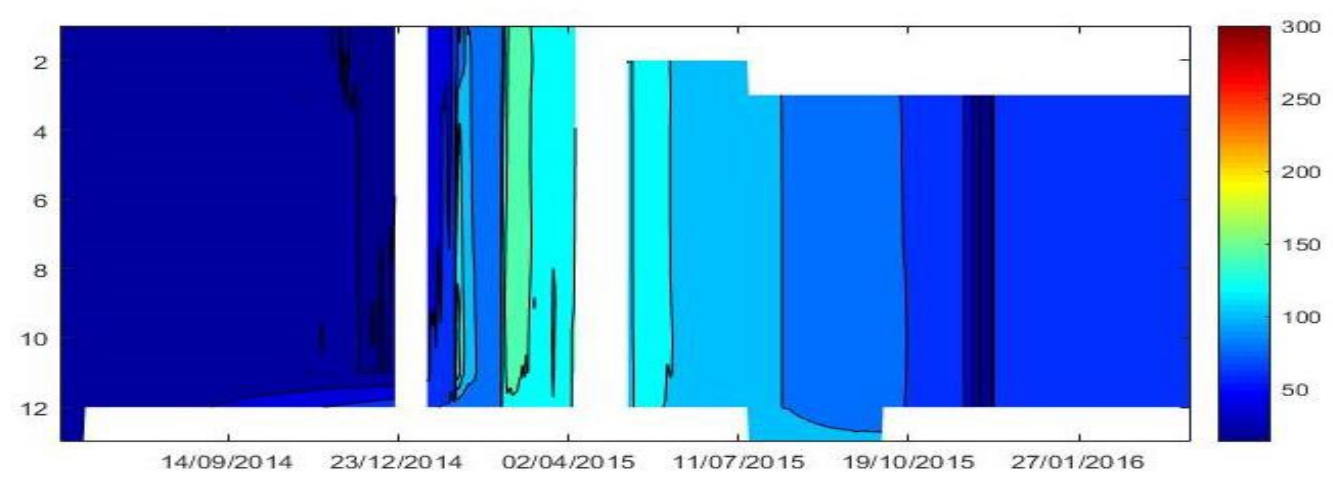

Figure 4: Raw fDOM readings (RFU), Tingalpa Reservoir 2014-2016. Left-hand y axis: depth [m]. 


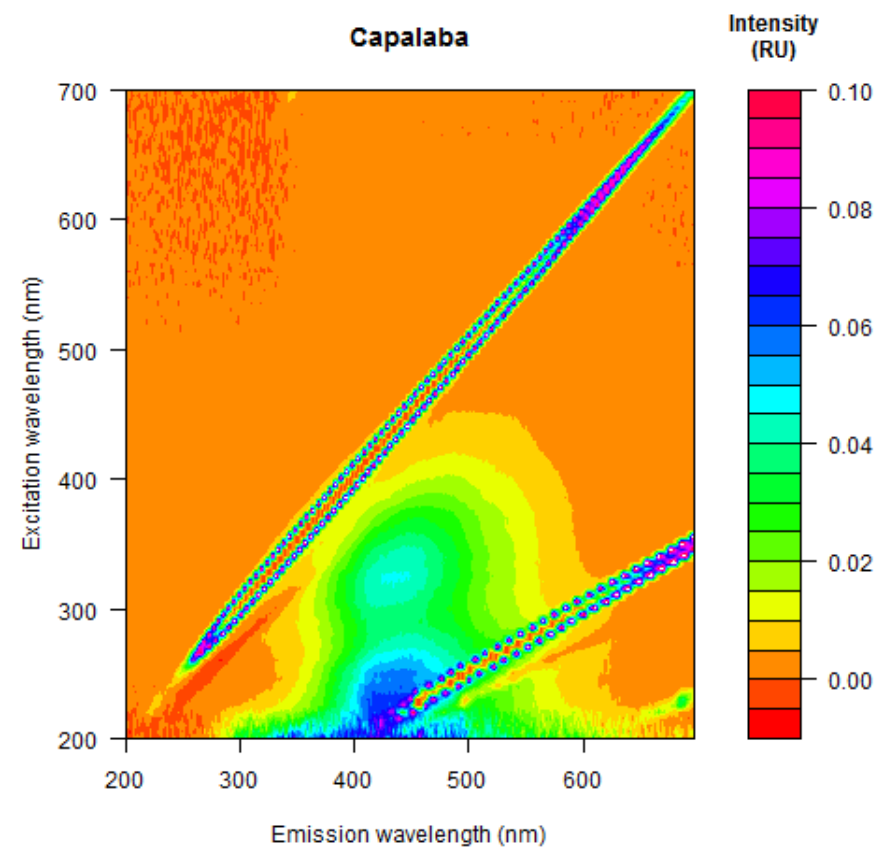

Figure 5: EEM for Tingalpa Reservoir water sample

From the results of the HPSEC, it was noticed that the bulk of the DOM had a MW between $500 \mathrm{Da}$ and $1500 \mathrm{Da}$, thus overall indexes such as DOC, $\mathrm{UV}_{254}$ and to some extent fDOM showed a good correlation $\left(\mathrm{R}^{2}>0.9\right)$ with this MW fraction. However, interestingly, normalised fDOM readings were also strongly, negatively correlated with low MW fractions. In particular, a very strong $\left(\mathrm{R}^{2}>0.99\right)$ correlation was found with the fraction of MW $<500 \mathrm{Da}$. This low MW DOM fraction has been previously reported to be extremely recalcitrant to removal by coagulation (Chow, Fabris, Leeuwen, Wang, \& Drikas, 2008) and thus it would very beneficial for a WTP to quantify in near-real time this specific DOM fraction.

\section{CONCLUSIONS}

A sequential data-driven model has been developed to compensate the initial readings of an fDOM probe for trivial quenching influences. The model can accurately and reliably correct the raw fDOM readings for water temperature, turbidity and IFE interferences, and significantly improved measurement accuracy for a test set of data. The sequential model was shown to outperform other developed linear and non-linear multivariate regression models. The model was developed for Tingalpa Reservoir, in SEQ, Australia. Although work towards building a generalised compensation model has been conducted, at this stage it is recommended to develop site-specific compensation models, due to varying properties among different water turbidity and organic matter. Similarly, preliminary work has been conducted to compensate the chl-a and PC readings of the total algae probe for similar interferences, as well as environmental conditions affecting the cellular pigment content by cyanobacteria. The sequential fDOM compensation model was used to compare compensated fDOM readings with specific organic matter properties, based on results of EEM and HPSEC experiments on different raw water sources. In general, fDOM readings measure reliably 
humic substances, which have been previously linked to increased metal toxicity at low $\mathrm{pH}$ (Holland, Wood, Smith, Correia, \& Val, 2017). However, it was also found that site-specific models can be developed to link compensated, normalized fDOM readings to different fractions of DOM (e.g. fulvic acids, in addition to humic acids). In addition, a strong correlation was found with low ( $<500 \mathrm{Da})$ molecular weight DOM, i.e. the DOM fraction more recalcitrant to removal by coagulation. Although more data needs to be collected to validate findings presented in this paper, there seems to be potential to link fDOM readings to DOM removal ability; this could lead to improving existing coagulant dosing prediction models (Bertone, Stewart, Zhang, \& O'Halloran, 2016; Kastl, Sathasivan, Fisher, \& Van Leeuwen, 2004) based on specific information on the fraction that is recalcitrant to removal, and by making the model rely mainly on real-time remote data. This practical outcome would enhance the capability of WTP operators to proactively mitigate the adverse effects of incoming raw water with high and/or previously unknown DOM characterisation. Moreover, there is also the potential to develop a model indicating the likelihood of an abundance of different cyanobacteria species based on only real-time remote sensing data, leading to a more proactive management of toxic blooms and taste and odour events.

\section{Acknowledgements}

The authors would like to thank Griffith University, University of South Australia, Seqwater and Xylem Analytics Australia for providing technical and financial support for this project. It is also acknowledged the partial contribution of Ms. Xinchen Wang (Griffith University), who performed a small percentage of the data analysis.

\section{References}

Bertone, E., Burford, M. A., \& Hamilton, D. P. (2018). Fluorescence probes for real-time remote cyanobacteria monitoring: a review of challenges and opportunities. Water Research, 141, 152-162.

Bertone, E., \& O'Halloran, K. (2016). Analysis and Modelling of Taste and Odour Events in a Shallow Subtropical Reservoir. Environments, 3(3), 22.

Bertone, E., Stewart, R. A., Zhang, H., \& O'Halloran, K. (2016). Hybrid water treatment cost prediction model for raw water intake optimization. Environmental Modelling \& Software, 75, 230242. doi: http://dx.doi.org/10.1016/j.envsoft.2015.10.020

Beutler, M., Wiltshire, K. H., Arp, M., Kruse, J., Reineke, C., Moldaenke, C., \& Hansen, U. P. (2003). A reduced model of the fluorescence from the cyanobacterial photosynthetic apparatus designed for the in situ detection of cyanobacteria. Biochimica et Biophysica Acta (BBA) Bioenergetics, 1604(1), 33-46. doi: http://dx.doi.org/10.1016/S0005-2728(03)00022-7

Brient, L., Lengronne, M., Bertrand, E., Rolland, D., Sipel, A., Steinmann, D., . . . Bormans, M. (2008). A phycocyanin probe as a tool for monitoring cyanobacteria in freshwater bodies. Journal of Environmental Monitoring, 10(2), 248-255.

Carstea, E. M. (2012). Fluorescence spectroscopy as a potential tool for in-situ monitoring of dissolved organic matter in surface water systems: INTECH Open Access Publisher.

Chang, D.-W., Hobson, P., Burch, M., \& Lin, T.-F. (2012). Measurement of cyanobacteria using in-vivo fluoroscopy-Effect of cyanobacterial species, pigments, and colonies. Water Research, 46(16), 5037-5048.

Chen, W., Westerhoff, P., Leenheer, J. A., \& Booksh, K. (2003). Fluorescence Excitation-Emission Matrix Regional Integration to Quantify Spectra for Dissolved Organic Matter. Environmental Science \& Technology, 37(24), 5701-5710. doi: 10.1021/es034354c 
Chow, C. W., Fabris, R., Leeuwen, J. v., Wang, D., \& Drikas, M. (2008). Assessing natural organic matter treatability using high performance size exclusion chromatography. Environmental science \& technology, 42(17), 6683-6689.

Downing, B. D., Pellerin, B. A., Bergamaschi, B. A., Saraceno, J. F., \& Kraus, T. E. C. (2012). Seeing the light: The effects of particles, dissolved materials, and temperature on in situ measurements of DOM fluorescence in rivers and streams. Limnology and Oceanography: Methods, 10(10), 767-775. doi: 10.4319/lom.2012.10.767

Henrion, R., Henrion, G., Böhme, M., \& Behrendt, H. (1997). Three-way principal components analysis for fluorescence spectroscopic classification of algae species. Fresenius' Journal of Analytical Chemistry, 357(5), 522-526.

Holland, A., Wood, C. M., Smith, D. S., Correia, T. G., \& Val, A. L. (2017). Nickel toxicity to cardinal tetra (Paracheirodon axelrodi) differs seasonally and among the black, white and clear river waters of the Amazon basin. Water Research, 123(Supplement C), 21-29. doi: https://doi.org/10.1016/j.watres.2017.06.044

Kastl, G., Sathasivan, A., Fisher, I. A. N., \& Van Leeuwen, J. (2004). Modeling DOC Removal by Enhanced Coagulation. Journal (American Water Works Association), 96(2), 79-89.

Pobel, D., Robin, J., \& Humbert, J.-F. (2011). Influence of sampling strategies on the monitoring of cyanobacteria in shallow lakes: Lessons from a case study in France. Water Research, 45(3), 10051014.

Watras, C. J., Hanson, P. C., Stacy, T. L., Morrison, K. M., Mather, J., Hu, Y. H., \& Milewski, P. (2011). A temperature compensation method for CDOM fluorescence sensors in freshwater. Limnology and Oceanography: Methods, 9(7), 296-301. doi: 10.4319/lom.2011.9.296

Zamyadi, A., Choo, F., Newcombe, G., Stuetz, R., \& Henderson, R. K. (2016). A review of monitoring technologies for real-time management of cyanobacteria: Recent advances and future direction. TrAC Trends in Analytical Chemistry, 85, 83-96.

Ziegmann, M., Abert, M., Müller, M., \& Frimmel, F. H. (2010). Use of fluorescence fingerprints for the estimation of bloom formation and toxin production of Microcystis aeruginosa. Water Research, 44(1), 195-204. 